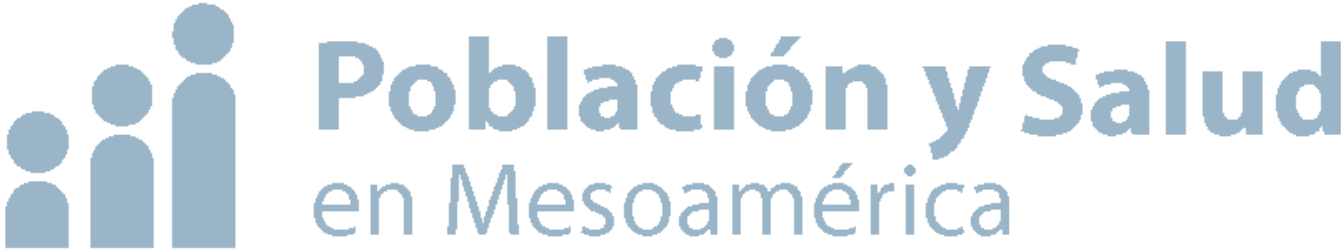

\section{Ensayo de micronúcleos con bloqueo de la citocinesis como biomarcador de daño genético en poblaciones sobreexpuestas a radiaciones ionizantes}

Andrés Cháves-Campos, Luisa Valle Bourrouet, Wendy MalespínBendaña y Vanessa Ramírez-Mayorga

\section{Como citar este artículo:}

Chaves-Campos, A., Valle-Bourrouet L., Malespín-Bendaña, W. y Ramírez-Mayorga, V. (2022). Ensayo de micronúcleos con bloqueo de la citocinesis como biomarcador de daño genético en poblaciones sobreexpuestas a radiaciones ionizantes. Población y Salud en Mesoamérica, 19(2). Doi: 10.15517/psm.v0i19.48078

\section{@)(1) (2)}

ISSN-1659-0201 http://ccp.ucr.ac.cr/revista/

Revista electrónica semestral Centro Centroamericano de Población Universidad de Costa Rica 


\title{
Ensayo de micronúcleos con bloqueo de la citocinesis como biomarcador de daño genético en poblaciones sobreexpuestas a radiaciones ionizantes
}

\author{
Micronucleus assay as biomarker of DNA damage in population exposed to ionizing \\ radiation
}

Andrés Cháves-Campos ${ }^{1}$, Luisa Valle Bourrouet ${ }^{2}$, Wendy Malespín-Bendaña ${ }^{3}$ y Vanessa Ramírez-Mayorga ${ }^{4}$

Resumen: Introducción: las radiaciones ionizantes (RI) son capaces de perjudicar el ADN; para evaluar este fenómeno es posible utilizar la formación de micronúcleos como biomarcador de efecto temprano del daño radioinducido. El ensayo de micronúcleos con bloqueo de la citocinesis (MNBC) es una técnica citogenética que permite demostrar el impacto de agentes genotóxicos. Propósito: en el presente trabajo se describieron mecanismos moleculares involucrados en la radioinducción de micronúcleos, la técnica del MNBC, los criterios de análisis, sus aplicaciones dentro de la investigación biológica y su extensión a la clínica, con énfasis en su empleo como biomarcador del daño genético en grupos sobreexpuestos a RI. Argumentos para la discusión: el MNBC se considera un método confiable, simple y rápido y existe evidencia de su aplicabilidad para el estudio de los efectos biológicos en casos de riesgo ocupacional y en accidentes radiológicos aislados o a gran escala. Conclusiones: el MNBC es una herramienta valiosa que posibilita estimar las consecuencias por dosis bajas de RI en poblaciones involucradas y, a la vez, orientar la toma de decisiones en cuanto a su prevención o atenuación. De igual forma, puede ser utilizado en análisis del campo de la radiobiología, a fin de detallar las incidencias de las radiaciones ionizantes sobre el ADN.

Palabras claves: micronúcleos, bloqueo de la citocinesis, biomarcadores de genotoxicidad, radiaciones ionizantes.

Abstract: Introduction. Ionizing radiation (IR) is capable of causing DNA damage. For the evaluation of this phenomenon it is possible to use chromosomal aberrations as biomarkers. The "Cytokinesis-Block Micronucleus assay" (CBMN) is a cytogenetic technique that allows to demonstrate the effect of genotoxic agents. Proposition: In the present review, we will describe the molecular mechanisms involved in micronucleus radioinduction, the micronucleus technique and criteria for analysis, its applications within biological research and its extension in clinical research, with emphasis on its application as a biomarker of radioinduced genetic damage. Arguments for discussion: the CBMN is considered a reliable, simple and fast technique and there is evidence of its applicability in the evaluation of biological effects in occupationally exposed personnel and in isolated or large-scale radiological accidents. Conclusions: the CBMN a valuable tool in estimating radiological risk in populations exposed to low doses of IR, allowing to guide decision-making regarding prevention or mitigation of exposure to IR in populations involved. Similarly, the cbmn can be used in research in the field of radiobiology, as a means to describe the effects of ionizing radiation on DNA.

Key words: micronuclei, cytokinesis-block, genotoxicity biomarkers, ionizing radiation.

Recibido:15 ago, 2021| Corregido:12 oct, 2021| Aceptado:10 nov, 2021

\footnotetext{
${ }^{1}$ Universidad de Costa Rica, San José, COSTA RICA. Correo: fabio.chavescampos@ucr.ac.cr ORCID https://orcid.org/0000-0003-3066-5495

2 Universidad de Costa Rica, San José, COSTA RICA. Correo: luisa.valle@ucr.ac.cr ORCID https://orcid.org/0000-0002-8621-1604

${ }^{3}$ Universidad de Costa Rica, San José, COSTA RICA. Correo: wendy.malespin@ucr.ac.cr ORCID https://orcid.org/0000-0002-1466-4815

${ }^{4}$ Universidad de Costa Rica, San José, COSTA RICA. Correo: vanessa.ramirez@ucr.ac.cr ORCID https://orcid.org/0000-0003-4104-3261
} 


\section{Introducción}

Los biomarcadores son parámetros biológicos que brindan información sobre el estado fisiológico o patológico de un individuo o población (Strimbu y Tavel, 2010). Entre los existentes, la frecuencia de micronúcleos (MN) es ampliamente utilizada en estudios de genotoxicidad básica y aplicada, incluyendo el área clínica, el biomonitoreo ocupacional y la dosimetría biológica (DB) (Torres-Bugarín et al., 2014).

Los MN son formaciones extranucleares y contienen fragmentos cromosómicos acéntricos o cromosomas (chrs) no incorporados en el núcleo de alguna de las células hijas luego de la citocinesis. Se encuentran envueltos por su propia membrana, por lo que toman la apariencia similar a la de un núcleo de un menor tamaño. Agentes genotóxicos, como las radiaciones ionizantes (RI), son capaces de inducir la formación de MN (Luzhna et al., 2013).

Fenech y Morley (1985) describieron por primera vez el ensayo de micronúcleos con bloqueo de la citocinesis (MNBC), este permite la identificación de células binucleadas con MN, las cuales se han dividido una vez. Las células son tratadas con citocalasina $B$, agente inhibidor del ensamblaje de microfilamentos de actina del anillo contráctil, a fin de facilitar la cariocinesis e inhibir la citocinesis. Para esta técnica citogenética es común obtener muestras de linfocitos periféricos, células epiteliales de mucosa bucal o nasal y fibroblastos (Falck, 2014).

El MNBC ha sido muy reconocido en el biomonitoreo de individuos expuestos a RI, pues esta es capaz de infligir daño sobre el ADN, lo que conlleva un riesgo de salud a corto y largo plazo. La vigilancia de tal factor genotóxico en el personal ocupacionalmente expuesto (POE) contribuye a adoptar medidas preventivas de radioprotección y de buenas prácticas radiológicas; en ese sentido, el MNBC constituye un valioso biomarcador de efecto temprano en esquemas de salud ocupacional del POE (Pajic et al., 2017).

A continuación, se describirán los mecanismos moleculares involucrados en la radioinducción de micronúcleos, la técnica de micronúcleos y sus criterios de análisis, las aplicaciones del ensayo dentro de la investigación biológica y su extensión a la investigación clínica, con énfasis en su uso como biomarcador del daño genético radioinducido.

\section{Desarrollo}

\subsection{Riesgo radiológico}

En la célula, el daño radioinducido al ADN se clasifica como letal o subletal. El primero es irreparable, compromete de forma irreversible la funcionalidad o viabilidad celular, por lo que lleva a la muerte de la célula durante la interfase o imposibilita la división (Sacristán, 2015); mientras tanto, el segundo puede tener tres posibles desenlaces (Giaccia et al., 2012):

1. Reparación completa del daño, que lleva a la supervivencia.

2. Reparación incompleta del daño; las modificaciones serán retenidas en el genoma celular y heredadas a la progenie celular.

3. No reparación del daño; varias lesiones subletales acumulativas pueden derivar en un daño no reparable de tipo letal. 
Según la naturaleza de la lesión producida, los efectos biológicos se catalogan en estocásticos y determinísticos. Por una parte, en los estocásticos, se considera que no existe un umbral de dosis de exposición a RI para su ocurrencia, por lo que el daño en una sola célula irradiada sobreviviente con una modificación en su genoma tiene potencial de transformación maligna. La probabilidad de que un individuo padezca de alguna patología como el cáncer aumenta de manera proporcional a la dosis de RI a la que es expuesto, comportamiento conocido como el modelo lineal sin umbral o LNT por sus siglas en inglés (linear no-threshold model). Este modelo de estimación de los efectos radioinducidos es el predominante en la práctica de la protección radiológica y se utiliza como base para estructurar programas y prácticas de seguridad (International Atomic Energy Agency, 2010).

Por otra parte, los determinísticos se producen por la muerte de un número elevado de células, lo cual se asocia a una pérdida de función, conforme al tejido u órgano afectado, la dosis y el volumen irradiado. Para su surgimiento, se requiere de un umbral mínimo de dosis de exposición (Huda, 2016); además, la severidad del daño aumenta con la cantidad de radiación (International Atomic Energy Agency, 2010). La respuesta clínica final a un valor por encima del umbral varía con base en la región o el órgano irradiado. Por ejemplo, en hombres, el sometimiento de las gónadas a 0.1 Sv produce esterilidad temporal (depleción de las espermatogonias y cese de la espermatogénesis, cuyo menoscabo es dependiente de dosis) y permanente, cuando es de 3.6 Sv. En la piel, 2 Sv causan descamación, mientras que, 25 Sv, necrosis (Cronenwett y Johnston, 2018; Joiner, 2010; Okada y Fujisawa, 2019).

Entonces, las posibles consecuencias en la salud por exposición a RI están supeditadas a la dosis a las que los individuos son expuestos. Los resultados de altas medidas de RI (aquellas por encima de 100 mGy) se encuentran bien documentados (Averbeck, 2009; International Atomic Energy Agency, 2010), sin embargo, a bajas magnitudes (por debajo del límite de 20-100 mSv/año) la relación dosis-efecto se vuelve más compleja de describir (Hall et al., 2017; Vaiserman, 2010). Las cifras de radiación involucradas en radiología diagnóstica son raramente suficientes como para vincularse con efectos determinísticos. A estos bajos niveles, los riesgos radiológicos se circunscriben a efectos estocásticos, esencialmente de tipo somático, en particular representados por un incremento a largo plazo en la incidencia de cáncer (Huda, 2016; Cardarelli y Ulsh, 2018).

El daño subletal puede evidenciarse como MN, donde las células radioinducidas tienen la capacidad de proliferar, sin embargo, durante la anafase se produce una mala segregación de los cromosomas o de fragmentos acéntricos; por eso, los MN son biomarcadores que representan eventos tempranos radioinducidos y estos, a largo plazo, acarrean un mayor riesgo de cáncer.

\subsection{Inducción de micronúcleos por radiaciones ionizantes}

Si bien las RI perjudican biomoléculas como lípidos y proteínas, el ADN es la diana principal con la cual producen su efecto en la célula, mediante distintos tipos de daño a la molécula de ADN, por ejemplo, rupturas monocatenarias, rupturas bicatenarias, alteraciones de bases nitrogenadas, entrecruzamiento entre ADN y proteínas de la cromatina. También, modificaciones en cromosomas, como la formación de 
cromosomas dicéntricos, anillos céntricos, fragmentos acéntricos, translocaciones y MN (Kavanagh et al., 2013; Organismo Internacional de Energía Atómica, 2014).

Los agentes capaces de inducir MN, según su mecanismo de acción, se dividen en clastogénicos o aneugénicos. Los clastógenos (como las RI) impulsan la formación de MN que incluyen fragmentos acéntricos o cromatídicos (Kisurina-Evgenieva et al., 2016). En tanto, los agentes aneugénicos (como la vincristina) llevan a MN con cromátidas o cromosomas completos (Fenech et al., 2011; Kirsh-Volders et al., 2011; Sabharwal et al., 2015; Kisurina-Evgenieva et al., 2016). Por medio del uso de sondas pancentroméricas en el MNBC, es posible discriminar entre MN con o sin centrómero (Vral et al., 2011), a fin de determinar el tipo de agente que originó el daño.

\subsubsection{Rupturas de hebra simple y de doble hebra de ADN}

La RI produce rupturas de hebra simple (RSH) y de doble hebra (RDH), a través de la deposición directa de energía del fotón o partícula en los átomos constituyentes de la molécula de ADN. No obstante, ocurre con mayor frecuencia la formación de rupturas a partir de un efecto indirecto, producto de la interacción de especies reactivas del oxígeno (principalmente radicales $\bullet \mathrm{OH}$ resultantes de la radiólisis del agua) con el ADN, que rompe los enlaces fosfodiéster (Giaccia et al., 2012; Kavanagh et al., 2013).

Se considera que el mecanismo básico en el surgimiento de MNs inducidos de forma directa o indirecta por la RI involucra la formación de fragmentos acéntricos con la inducción de RDH. Así, la célula inicia una respuesta coordinada al daño radioinducido al ADN e incluye una serie de mecanismos dirigidos a activar puntos de control del ciclo celular, reparar el daño y dar señales de sobrevivencia o apoptosis (Joiner, 2010). Con todo, a pesar de que las vías de respuesta al daño radioinducido al ADN posibilitan resolver gran parte del daño inicial, algunas células con RDH continúan hacia la mitosis. La presencia de $\mathrm{RDH}$ en una misma molécula produce fragmentos acéntricos, los cuales no segregarán correctamente durante la anafase y conducen a la aparición de MN (Fenech et al., 2011).

La presencia de RSHs puede derivar en RDHs si no son reparadas correctamente por escisión de bases (BER), por tanto, tienen un papel relevante en la inducción de MN (Khoronenkova y Dianov, 2015). En concreto, si una horquilla de replicación se encuentra con una RSH durante la fase S, puede desencadenar el colapso de la primera y la consecuente RDH (Joiner y van der Kogel, 2019). De igual forma, la coincidencia de dos RSH a una distancia de hasta 10 pares de bases podría devenir en una RDH e interrumpir la continuidad de la molécula de ADN (Schipler y lliakis, 2013).

\subsubsection{Reparación inadecuada de rupturas de la molécula de ADN}

Las RDHs son reparadas por dos vías principales: la unión de extremos no homólogos (UENH) y la recombinación homóloga $(\mathrm{RH})$. El mecanismo de reparación de $\mathrm{RDH}$ radioinducidas depende, en primera instancia, de la fase del ciclo celular de la célula expuesta. En células de mamíferos, la UENH repara $\mathrm{RDH}$ a lo largo del ciclo celular, mientras que, la $\mathrm{RH}$ solo participa en las fases S/G2 (Shibata, 2017). Al contemplar que células como los linfocitos periféricos se encuentran generalmente en un 
estado de quiescencia (G0) (Organismo Internacional de Energía Atómica, 2014), el principal mecanismo de reparación de RDH es la UENH canónica e intervienen en menor medida otros como la RH y las vías de la UENH alternativas (estas últimas en humanos parecen no contribuir de forma significativa durante las fases G0/G1) (figura 1) (Forster et al., 2019).

Figura 1

Mecanismo general de formación de micronúcleos radioinducidos

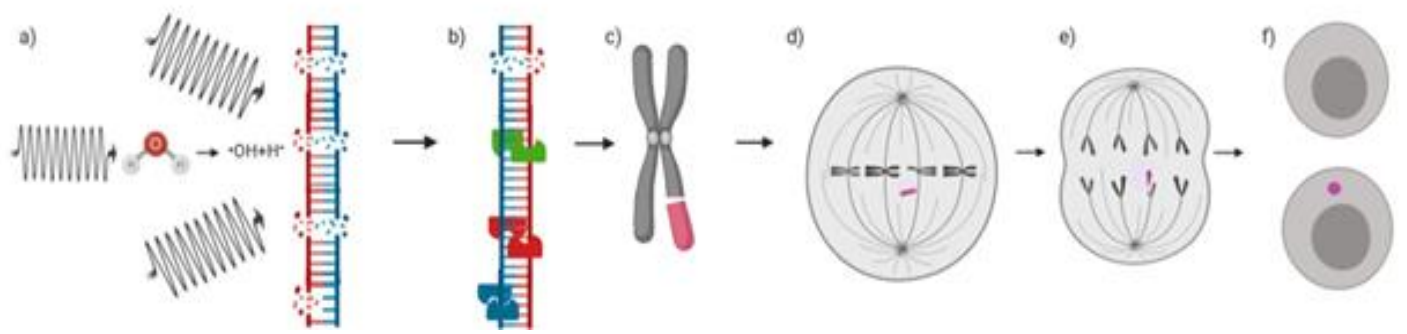

Nota. a) Las RI (líneas negras onduladas) producen SSB (puntos rojos) y DSB (puntos rojos y azules) a través de un efecto directo y uno indirecto mediado por radicales libres. b) SSB es reparado por escisión de bases (BER) (enzimas azules) y las DSB, principalmente por UENH (enzimas rojas) y HR (enzimas verdes), algunos daños permanecen sin ser reparados (puntos rojos y azules). c) La presencia de DSB puede formar fragmentos acéntricos cromatídicos o cromosómicos (color rosa). d) Aquellos fragmentos acéntricos (color rosa) que persistan hasta la mitosis no serán capaces de interactuar con los microtúbulos del huso mitótico. e) Durante la anafase, estos fragmentos (color rosa) quedarán rezagados, en tanto que, por acción de la maquinaria de segregación, el resto del material cromosómico se dirige a polos opuestos del huso mitótico. f) En la telofase, el surgimiento de nuevas membranas nucleares da paso a núcleos hijos, cada uno con una copia del material genético completo, excepto los fragmentos acéntricos rezagados, los cuales serán rodeados por su propia membrana, conduciendo a la formación de micronúcleos (núcleo de menor tamaño de color rosa).

En células de mamíferos, la vía UENH canónica requiere del heterodímero Ku (Ku70/80), el cual reconoce la ruptura y recluta a la subunidad catalítica de la proteína quinasa dependiente de ADN; este complejo se une a ambos extremos de la ruptura y, a su vez, recluta la nucleasa Artemis, que procesa los extremos dañados, haciéndolos compatibles y ligables; posteriormente, los extremos serán unidos por la ligasa IV/XRCC4/XLF. Este mecanismo es susceptible a errores, pues la ruptura no es reparada utilizando un molde homólogo y, en consecuencia, se pueden producir deleciones, inserciones y translocaciones (Kavanagh et al., 2013; Rassool y Tomkinson, 2010). Por acción de la UENH se pueden evitar en parte MNs, pero pueden formarse otros tipos de aberraciones cromosómicas no detectadas por el MNBC.

Al respecto, se han observado polimorfismos en genes de sistemas de reparación de ADN (XRCC3, XRCC1, XPD) que parecen contribuir en la susceptibilidad genética en POE a bajas dosis de radiación, esto incrementa la frecuencia de MN con respecto a grupos control (Angelini et al., 2005; Cho et al., 2009; Kavanagh et al., 2013). 
Durante la trayectoria de los fotones o partículas de RI a través del núcleo celular, son posibles múltiples eventos de radiólisis en moléculas de agua, cada una de las especies reactivas resultantes ocasionan daño oxidativo en el ADN a manera de lesiones agrupadas (en una cercanía menor a 20 pares de bases o de menos de dos giros de la doble hélice de ADN). Intentos de reparación simultáneos por BER del daño oxidativo agrupado en hebras opuestas podrían formar $\mathrm{RDH}$ y fragmentos acéntricos que luego serían incluidos en MNs (Cannan et al., 2014; Cannan y Pederson, 2016).

El acceso de las especies reactivas del oxígeno radioinducidas a los posibles sitios de daño está determinado por la geometría de la molécula de ADN y la compactación de la cromatina. Además, la transferencia lineal de energía de la RI (LET, cantidad de energía depositada por la RI unidad de distancia) establece el patrón de distribución espacial de las rupturas radioinducidas (Carter et al., 2018; Kavanagh et al., 2013; Nickoloff et al., 2013; Nikitaki et al., 2016; Zhang et al., 2016). Las RI con bajo LET (rayos $\mathrm{X}$, rayos gamma, partículas beta) inducen menor cantidad de eventos de ionización y, por ende, lesiones aisladas del ADN. Al contrario, las RI con alto LET (partículas alfa, protones) al producir una mayor cantidad de eventos de ionización, aumentan las lesiones agrupadas y los fragmentos de ADN de reducida longitud (Nickoloff et al., 2013; Lavelle y Foray, 2014). Se sabe que las lesiones agrupadas son reparadas con menor eficacia, este comportamiento se explica en parte por la inhibición de la holoenzima DNA-PK cuando se une a fragmentos cortos de ADN, causando el bloqueo de la ejecución del mecanismo de UENH canónica; al bloquearse esta vía la reparación recae sobre los mecanismos de HR y UENH alternativa (Nickoloff et al., 2013). Debido a que el aporte de estos dos últimos mecanismos en la reparación del $A D N$ en las fases $G 0 / G 1$ es menor que en otras fases, es esperable que las lesiones agrupadas se reparen en menor proporción que las lesiones simples aisladas y ello favorece la formación de MNs.

\subsubsection{Mecanismos secundarios de formación de micronúcleos}

La principal modalidad en la formación de MNs inducidos de forma directa o indirecta por la RI es a través del escape a los mecanismos de reparación celular de RDH y RSH, lo que lleva a la producción de fragmentos acéntricos que quedan rezagados en la anafase. Sin embargo, existen otros mecanismos que podrían contribuir en menor medida, tales como la cromotripsis (Antonin y Neumann, 2016; Cannan y Pederson, 2016; Maher y Wilson, 2012; Morishita et al., 2016), la ruptura de cromosomas dicéntricos y la inestabilidad genética (Fenech et al., 2011; Sommer et al., 2020). Asimismo, factores independientes como la edad, el sexo, el consumo de alcohol y algunos agentes aneugénicos pueden influir en la frecuencia de MNs (Fenech et al., 2011; Luzhna et al., 2013; Sommer et al., 2020).

\subsection{Ensayo de micronúcleos con bloqueo de la citocinesis}

Los MNs, como biomarcadores de exposición a agentes genotóxicos, pueden ser valorados en linfocitos periféricos y en muestras de células exfoliadas de mucosa oral, bucal y urotelial (Falck, 2014). En el primer caso, se utiliza generalmente el método de bloqueo de la citoquinesis con citocalasina-B; debido a la acción de este agente, las células que hayan experimentado división celular solo una vez se 
caracterizarán por ser binucleadas. Los MNs son aberraciones cromosómicas inestables, el registro del biomarcador en solo células binucleadas evita la posibilidad de subestimar la frecuencia por pérdida de MNs en células con múltiples ciclos celulares (Fenech et al., 2003)

El Organismo Internacional de Energía Atómica (2014) propuso el protocolo estándar de MNCB, basado en el de Fenech y Morley (1985). El Proyecto Colaborativo Internacional en la Frecuencia de Micronúcleos en Poblaciones Humanas (Proyecto HUMN) ha publicado numerosas investigaciones sobre las aplicaciones de este ensayo en el análisis de distintas problemáticas de la salud humana y ha brindado una descripción detallada sobre los criterios para el conteo y el registro de células binucleadas con MN en linfocitos de sangre periférica (Fenech et al., 2003; Lyulko et al., 2014; Rodrigues et al., 2016); estos se enumeran a continuación:

1.Las células deben ser binucleadas, sus límites citoplasmáticos deben estar intactos y claramente distinguibles de los límites de otras células.

2.Ambos núcleos deben ser similares en tamaño y presentar membranas nucleares intactas que deben estar situadas dentro de los mismos límites citoplasmáticos.

3.El diámetro de los MN varía entre 1/16 y 1/3 del diámetro de uno de los núcleos principales.

4.Los MN son redondos u ovalados.

5.Los MN no son refráctiles, por lo que pueden diferenciarse de artificios (ej. partículas de tinción).

6.Los MN no están unidos o conectados con los núcleos principales.

7.Los MN pueden tocar, pero no traslaparse con los núcleos principales.

8.Los MN usualmente tienen la misma intensidad de tinción que los núcleos principales, en algunas ocasiones pueden teñirse con mayor intensidad (figura 2).

Figura 2

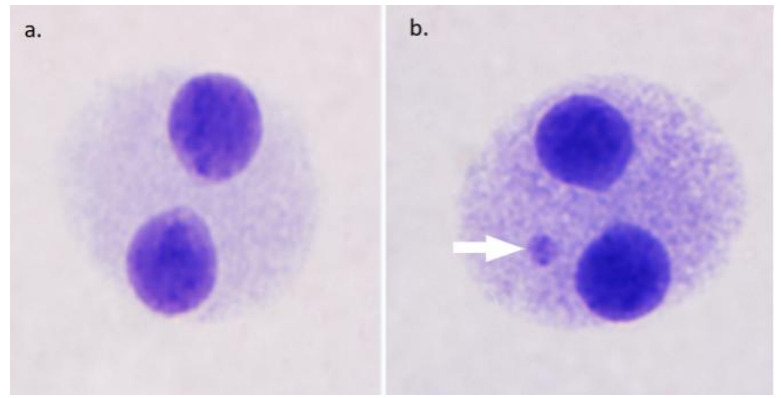

Nota. a) Célula binucleada sin presencia de micronúcleos. b) Célula binucleada con presencia de un micronúcleo (flecha blanca) (100x, INISA, 2018). 
El conteo de células binucleadas para el análisis de frecuencia de MN varía en virtud de diversos factores: cantidad de preparaciones hechas, tiempo disponible para efectuar el análisis, carga de trabajo o número de casos. Tanto para biomonitoreo como para DB se recomienda, al menos, contar 1000 células binucleadas (Palanikumar y Panneerselvam, 2011; Sullivan et al., 2013), aun así, es factible realizar el estudio bajo el criterio de triaje (gran cantidad de casos emergentes y número reducido de células analizadas), con al menos 500 células por exposición a rayos X hasta los 4.0 Gy (Rawojć et al., 2015). Dependiendo del diseño del estudio y del tipo de análisis estadístico, es posible aumentar el recuento de células binucleadas a 2000 (Castillo et al., 2011). De hecho, Rawojć et al. (2015) sostuvieron que no hay diferencia en los resultados producidos por distintos analistas entrenados, lo cual ejerce un impacto importante en los tiempos de respuesta.

El uso de métodos automatizados es más ventajoso frente al análisis manual de muestras (Willems et al., 2010), dado su alto rendimiento en términos de velocidad y objetividad (Shibai-Ogata et al., 2014). Permite emitir respuestas rápidas ante casos de triaje por accidentes radiológicos, sin embargo, debe contemplarse la posibilidad de introducir cambios en los métodos de tinción, por ejemplo, el yoduro de propidio en lugar de Giemsa, a fin de lograr resultados óptimos (Tamizh et al., 2015). La norma ISO 17099:2014 define los criterios para la aplicación del ensayo de MNBC en el ámbito de la dosimetría biológica, técnica que permite estimar dosis absorbida en individuos expuestos a RI en distintos escenarios de sobreexposición (Deminge et al., 2018).

\subsection{Biomonitoreo con el MNBC de los efectos de la exposición a RI}

El MNBC registra el daño radioinducido al material genético y ha sido muy aplicado en genotoxicidad básica y aplicada para evaluar el daño genético como consecuencia de riesgos ambientales u ocupacionales, por ello, constituye un instrumento auxiliar en la protección radiológica y uno fundamental en el campo de la radiobiología (Torres-Bugarín et al., 2014) y es una herramienta valiosa en la estimación del riesgo radiológico en el POE (International Commision on Radiological Protection, ICRP, 2011). Diversos estudios han mostrado que el personal en salud expuesto a dosis efectivas de radiación menores a los límites fijados por la ICRP ( $20 \mathrm{mSv} / \mathrm{año}$, promediada en un periodo de 5 años, sin que un solo año supere los $50 \mathrm{mSv}$ ) (ICRP, 2019) está sujeto a incrementos en la frecuencia de MN (tabla I).

Dicho método sirve a la dosimetría biológica en cuanto a estimaciones de dosis, donde es necesario conocer la relación (curva dosis-respuesta) entre la frecuencia del biomarcador y una dosis de exposición (Suto et al., 2015). Así, el MNBC es lo suficientemente sensible para calcular dosis en un rango de los 0.3 Gy a los 5 Gy e, incluso, con modificaciones hacia un enfoque multiparamétrico es posible alcanzar un rango de 0.1 Gy a $15 \mathrm{~Gy}$, para ello deben considerarse otros marcadores como los puentes nucleoplásmicos, brotes nucleares, células necróticas y apoptóticas y la valoración de la citotoxicidad mediante el conteo de células mononucleadas, binucleadas y polinucleadas (Rodrigues et al., 2018; Rodrigues et al, 2014). Tal ensayo permite detectar daño cromosómico hasta 6 meses después de la 
exposición (Alotaibi et al., 2018; Badie et al., 2016), así como en casos de exposición prolongada, reciente y aguda, irradiaciones parciales del cuerpo y de cuerpo entero (OIEA, 2014; Purnami et al., 2017))

De igual forma, el MNBC ha sido eficaz en evaluación de accidentes radiológicos a gran escala y en la detección de exposiciones graves ( $\geq 1 \mathrm{~Gy}$ ) que requieren de un tratamiento médico inmediato y oportuno. En este contexto, se utiliza la frecuencia de MN como herramienta complementaria a la evaluación clínica para estimar dosis, categorizar temprano a los individuos sobreexpuestos y seleccionar el manejo médico más apropiado para cada caso (OIEA, 2014); en estas situaciones, una respuesta pronta es esencial. En comparación con el ensayo de cromosomas dicéntricos, la técnica del MNBC es más sencilla y rápida, lo cual la convierte en una opción viable en casos de accidentes de exposición masiva. Al respecto, Rawojć et al. (2015) observaron que puede disminuirse la cantidad de células binucleadas a 500 y que múltiples analistas entrenados pueden participar en el registro de frecuencias, acciones que impactan en la disminución de tiempos de respuesta.

En suma, el uso de RI en distintas áreas del quehacer humano comprende una práctica cada vez más común, tanto en el área de atención en salud humana y animal como en la industria. El rol de los programas de seguridad radiológica es fundamental para garantizar el empleo seguro de las RI y proteger a las personas de los efectos adversos de la exposición. En este sentido, el MNBC ofrece un método útil para el monitoreo del daño al ADN producto de la exposición ocupacional a RI.

Tabla 1

Estudios de biomonitoreo de POE mediante el MNBC

\begin{tabular}{|c|c|c|c|c|c|c|c|}
\hline $\begin{array}{l}\text { Grupo/Característica } \\
\text { s }\end{array}$ & $\mathbf{N}$ & $\begin{array}{l}\text { Edad } \\
\text { promedi } \\
\text { o } \pm \text { DE } \\
\text { (años) }\end{array}$ & Área de trabajo & $\begin{array}{l}\text { Exposició } \\
\text { n (años) }\end{array}$ & MN & $\begin{array}{l}\text { Valo } \\
\text { rde } \\
\text { p }\end{array}$ & $\begin{array}{l}\text { Referenci } \\
\text { a }\end{array}$ \\
\hline \multicolumn{8}{|l|}{ Francia } \\
\hline No expuesto & 69 & $44.2 \pm 9.0$ & - & - & $\begin{array}{l}14.3 \pm \\
7.2^{\star}\end{array}$ & 0.011 & $\begin{array}{l}\text { (Sari- } \\
\text { Minodier }\end{array}$ \\
\hline Expuesto & $\begin{array}{l}13 \\
2\end{array}$ & $43.6 \pm 8.4$ & $\begin{array}{l}\text { Radioterapia, } \\
\text { medicina } \\
\text { nuclear, } \\
\text { radiología y } \\
\text { cardiología } \\
\text { intervencionista, } \\
\text { radiodiagnóstico }\end{array}$ & $16.5 \pm 8.5$ & $\begin{array}{l}17.0 \pm \\
9.9\end{array}$ & & $\begin{array}{l}\text { et al., } \\
2007)\end{array}$ \\
\hline \multicolumn{8}{|l|}{ Brasil } \\
\hline No expuesto & 22 & 32.5 & - & - & $\begin{array}{l}7.18 \pm \\
2.59\end{array}$ & 0.008 & $\begin{array}{l}\text { (Maluf et } \\
\text { al., 2001) }\end{array}$ \\
\hline Expuesto & 22 & 37.18 & $\begin{array}{l}\text { Radiodiagnóstic } \\
\text { o }\end{array}$ & $1-19$ & $\begin{array}{l}8.84 \pm \\
2.34\end{array}$ & & \\
\hline
\end{tabular}




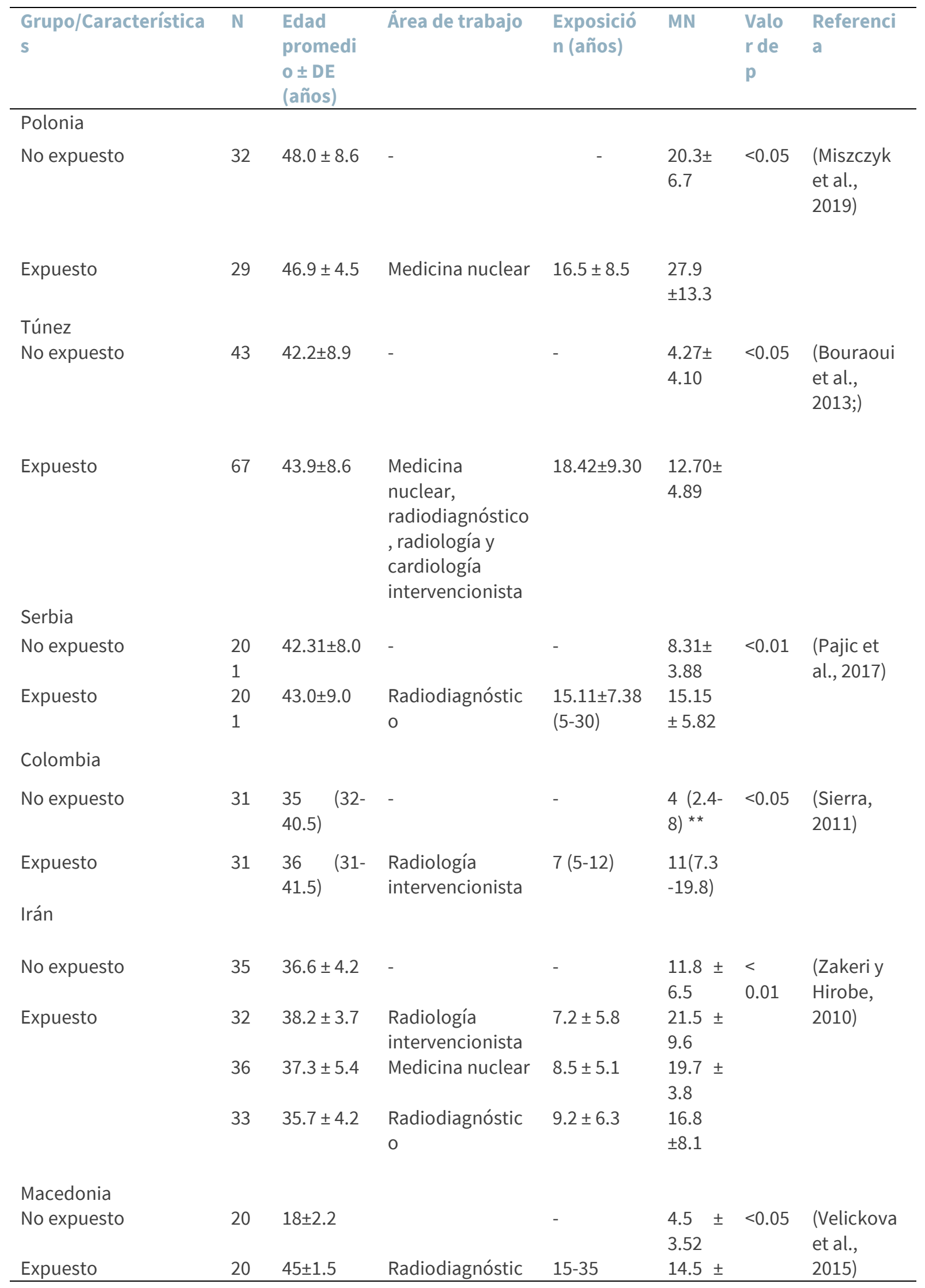




\begin{tabular}{|c|c|c|c|c|c|c|c|}
\hline $\begin{array}{l}\text { Grupo/Característica } \\
\text { s }\end{array}$ & $\mathrm{N}$ & $\begin{array}{l}\text { Edad } \\
\text { promedi } \\
\text { o } \pm \text { DE } \\
\text { (años) }\end{array}$ & Área de trabajo & $\begin{array}{l}\text { Exposició } \\
\text { n (años) }\end{array}$ & MN & $\begin{array}{l}\text { Valo } \\
\text { irde } \\
\text { p }\end{array}$ & $\begin{array}{l}\text { Referenci } \\
\text { a }\end{array}$ \\
\hline & & & 0 & & 5.51 & & \\
\hline
\end{tabular}

\section{Conclusiones y perspectivas}

El principal mecanismo mediante el cual las RI son capaces de inducir MN es la ruptura de la molécula de ADN, esto lleva a la formación de RDH y, posteriormente, a fragmentos acéntricos. Si tales daños no son reparados correctamente, las células prosiguen hacia la mitosis con fragmentos de ADN que no podrán interactuar con el huso mitótico. Luego, en la telofase, los fragmentos rezagados derivarán en la aparición de MN.

La sobreexposición a RI conlleva un importante riesgo para la salud, de ahí, es relevante el desarrollo de técnicas que permitan fortalecer los programas de protección radiológica. Dentro de las pruebas citogenéticas utilizadas en biomonitoreo ocupacional, el MNBC constituye una herramienta valiosa para estimar el riesgo radiológico a bajas dosis en el POE y orientar la toma de decisiones en cuanto a medidas preventivas o de atenuación ante la sobreexposición. De igual forma, sirve como medio a la investigación en el campo de la radiobiología para describir los efectos de las radiaciones ionizantes sobre el ADN y, en el clínico, para generar conocimiento acerca de la mejora de procedimientos radioterapéuticos.

Es factible aumentar la sensibilidad del MNBC para detectar daño radioinducido al ADN, usando sondas pancentroméricas, lo que facilita identificar el origen del MN entre aquellos formados a través de mecanismos clastogénicos y los formados por mecanismos aneugénicos. Con el análisis automatizado de MNs, se eleva el flujo de trabajo al disminuir el tiempo dedicado a cada caso, esto es especialmente importante en situaciones en donde existe gran cantidad de personas involucradas en sobreexposiciones accidentales.

Se ha demostrado que un elemento fundamental para garantizar la protección frente al uso de las RI en las distintas áreas del quehacer humano corresponde al establecimiento de técnicas de biomonitoreo con marcadores de daño al ADN, las cuales permiten detectar los efectos tempranos radioinducidos en el ámbito molecular y prevenir las consecuencias adversas de la sobreexposición con RI.

\section{Agradecimientos}


A la Dra. Isabel Castro-Volio, q.e.p.d., quien fue investigadora pionera de la citogenética y de la dosimetría biológica en Costa Rica. A Fernando Ortíz, Nelson Brenes y Alejandra Vargas, colaboradores del Laboratorio de Citogenética del INISA, su trabajo fue indispensable en la aplicación del ensayo de micronúcleos con bloqueo de la citoquinesis.

\section{Referencias}

Alotaibi, M., Alsuhaibani, E. y Alsbeih, G. (2018). Assessment of In vitro X-Ray Radiation Overexposure by Cytokinesis-Block Micronucleus Assay in Human Peripheral Blood Lymphocytes (HPBLs) in Saudi Population. Cellular and Molecular Biology, 64(1), 148. https://www.omicsonline.org/openaccess/assessment-of-in-vitro-xray-radiation-overexposure-by-cytokinesisblock-micronucleus-assayin-human-peripheral-blood-lymphocytes-hp-1165-158X-1000148-104159.html?view=mobile

Angelini, S., Kumar, R., Carbone, F., Maffei, F., Forti, G. C., Saverio Violante, F., Lodi, V., Curti, S., Hemminki, K., y Hrelia, P. (2005). Micronuclei in humans induced by exposure to low level of ionizing radiation: influence of polymorphisms in DNA repair genes. Mutation Research. https://doi.org/10.1016/j.mrfmmm.2004.10.007

Antonin, W. y Neumann, H. (2016). Chromosome condensation and decondensation during mitosis. Current Opinion in Cell Biology, 40, 15-22. https://doi.org/10.1016/j.ceb.2016.01.013

Averbeck, D. (2009). Does scientific evidence support a change from the LNT model for low-dose radiation nrisk extrapolation? Health Physics, 97(5), 493-504.

Badie, C., Hess, J., Zitzelsberger, H. y Kulka, U. (2016). Established and Emerging Biomarkers of Radiation Exposure. Clinical Oncology. https://doi.org/10.1016/j.clon.2016.06.002

Bouraoui, S., Mougou, S., Drira, A., Tabka, F., Bouali, N., Mrizek, N., Elghezal, H. y Saad, A. (2013). A cytogenetic approach to the effects of low levels of ionizing radiation (IR) on the exposed Tunisian hospital workers. International Journal of Occupational Medicine and Environmental Health, 26(1), 144154. https://doi.org/10.2478/s13382-013-0084-4

Cannan, W. J. y Pederson, D. S. (2016). Mechanisms and Consequences of Double-strand DNA Break Formation in Chromatin. J Cell Physiol, 231(1), 3-14. https://doi.org/0.1002/jcp.25048. Autho

Cannan, W. J., Tsang, B., Wallace, S. y Pederson, D. S. (2014). Nucleosomes Suppress the Formation of Double-strand DNA Breaks during Attempted Base Excision Repair of Clustered Oxidative Damages. J Biol Chem, 289(29), 19881-19893. https://doi.org/10.1074/jbc.M114.571588

Cardarelli, J. J. y Ulsh, B. A. (2018). It is time to move beyond the linear no-threshold theory for low-dose radiation protection. Dose-Response, 16(3). https://doi.org/10.1177/1559325818779651

Carter, R. J., Nickson, C. M., Thompson, J. M., Kacperek, A., Hill, M. A. y Parsons, J. L. (2018). Complex DNA Damage Induced by High Linear Energy Transfer Alpha-Particles and Protons Triggers a Specific Cellular DNA Damage Response. International Journal of Radiation Oncology Biology Physics, 100(3), 
776-784. https://doi.org/10.1016/j.ijrobp.2017.11.012

Castillo, E., Guevara-Fujita, M. L. y Fujita, R. (2011). Optimización del test de micronúcleos en linfocitos cultivados usando una metodología de gradiente y frotis. Revista Peruana de Biología.

Cho, Y. H., Kim, Y. J., An, Y. S., Woo, H. D., Choi, S. Y., Kang, C. M. y Chung, H. W. (2009). Micronucleuscentromere assay and DNA repair gene polymorphism in lymphocytes of industrial radiographers. Mutation Research - Genetic Toxicology and Environmental Mutagenesis. https://doi.org/10.1016/j.mrgentox.2009.08.007

Cronenwett, J. y Johnston, K. (2018). Rutherford's vascular surgery. (9th ed.). Elsevier.

Deminge, M., Radl, A., Taja, M., Fernández-Rearte, J., Cabitto, M. y Di Gior. (2018). Aplicabilidad del ensayo de micronúcleos - dosímetro biológico en escenarios con víctimas múltiples. Congreso Regional de La Asociación Internacional de Protección Radiológica.

Falck, G. (2014). Micronuclei in Human Peripheral Lymphocytes - Mechanistic Origin and Use as a Biomarker of Genotoxic Effects in Occupational Exposure.

Fenech, M., Chang, W. P., Kirsch-Volders, M., Holland, N., Bonassi, S. y Zeiger, E. (2003). HUMN project: Detailed description of the scoring criteria for the cytokinesis-block micronucleus assay using isolated human lymphocyte cultures. Mutation Research - Genetic Toxicology and Environmental Mutagenesis, 534(1-2), 65-75. https://doi.org/10.1016/S1383-5718(02)00249-8

Fenech, M., Kirsch-Volders, M., Natarajan, A. T., Surralles, J., Crott, J. W., Parry, J., Norppa, H., Eastmond, D. A., Tucker, J. D. y Thomas, P. (2011). Molecular mechanisms of micronucleus, nucleoplasmic bridge and nuclear bud formation in mammalian and human cells. Mutagenesis, 26(1), 125-132. https://doi.org/10.1093/mutage/geq052

Fenech, M. y Morley, A. (1985). Measurement of micronuclei in lymphocytes. Mutation Research/Environmental Mutagenesis and Related Subjects, 147(1), 29-36. https://doi.org/10.1016/0165-1161(85)90015-9

Forster, J. C., Douglass, M. J. J., Phillips, W. M. y Bezak, E. (2019). Stochastic multicellular modeling of x-ray irradiation, DNA damage induction, DNA free-end misrejoining and cell death. Scientific Reports, 9. https://doi.org/10.1038/s41598-019-54941-1

Giaccia, E., Hall, J. y Amato, J. (2012). Radiobiology for the radiologist. (7th ed.). Lippincott Williams \& Williams.

Hall, J., Jeggo, P. A., West, C., Gomolka, M., Quintens, R., Badie, C. Cardis, E. (2017). Ionizing radiation biomarkers in epidemiological studies - An update. In Mutation Research - Reviews in Mutation Research (Vol. 771, pp. 59-84). Elsevier B.V. https://doi.org/10.1016/j.mrrev.2017.01.001

Huda, W. (2016). Review of radiologic physics (4th ed.). Wolters Kluwer.

International Atomic Energy Agency. (2010). Radiation Biology: A Handbook for Teachers and Students. 
International Atomic Energy Agency (ed.).

International Commision on Radiological Protection. (2011). ICRP statement on Tissue Reactions [Archivo PDF]. http://www.icrp.org/docs/ICRP Statement on Tissue Reactions.pdf

International Commision on Radiological Protection. (2019). Dose limits. http://icrpaedia.org/Dose_limits

Joiner, M. y van der Kogel, A. (2019). Basic Clinical Radiobiology. (5th ed.). CRC Press.

Kavanagh, J., Redmond, K., Schettino, G. y Prise, K. (2013). DSB Repair - A radiation perspective. Antioxidants \& Redox Signaling, 18, 2458-2472.

Khoronenkova, S. V. y Dianov, G. L. (2015). ATM prevents DSB formation by coordinating SSB repair and cell cycle progression. Proceedings of the National Academy of Sciences. https://doi.org/10.1073/pnas.1416031112

Kirsch-Volders, M., Plas, G., Elhajouji, A., Lukamowicz, M., Gonzalez, L., Vande Loock, K. y Decordier, I. (2011). The in vitro MN assay in 2011: Origin and fate, biological significance, protocols, high throughput methodologies and toxicological relevance. Archives of Toxicology, 85(8), 873-899. https://doi.org/10.1007/s00204-011-0691-4

Kisurina-Evgenieva, O. P., Sutiagina, O. I. y Onishchenko, G. E. (2016). Biogenesis of micronuclei. Biochemistry (Moscow). https://doi.org/10.1134/S0006297916050035

Lavelle, C. y Foray, N. (2014). Chromatin structure and radiation-induced DNA damage: From structural biology to radiobiology. International Journal of Biochemistry and Cell Biology, 49, 84-97. https://doi.org/10.1016/j.biocel.2014.01.012

Luzhna, L., Kathiria, P. y Kovalchuk, O. (2013). Micronuclei in genotoxicity assessment: From genetics to epigenetics and beyond. Frontiers in Genetics, 4(JUL), 1-17. https://doi.org/10.3389/fgene.2013.00131

Lyulko, O. V., Garty, G., Randers-Pehrson, G., Turner, H. C., Szolc, B. y Brenner, D. J. (2014). Fast Image Analysis for the Micronucleus Assay in a Fully Automated High-Throughput Biodosimetry System. Radiation Research. https://doi.org/10.1667/RR13441.1

Maher, C. A. y Wilson, R. K. (2012). Chromothripsis and human disease: Piecing together the shattering process. In Cell. https://doi.org/10.1016/j.cell.2012.01.006

Maluf, S. W., Passos, D. F., Bacelar, A., Speit, G. y Erdtmann, B. (2001). Assessment of DNA damage in lymphocytes of workers exposed to X-radiation using the micronucleus test and the comet assay. Environmental and Molecular Mutagenesis, 38(4), 311-315. https://doi.org/10.1002/em.10029

Miszczyk, J., Rawojć, K., Panek, A., Gałaś, A., Kowalska, A., Szczodry, A. y Brudecki, K. (2019). Assessment of the nuclear medicine personnel occupational exposure to radioiodine. European Journal of Radiology, 121(October). https://doi.org/10.1016/j.ejrad.2019.108712 
Morishita, M., Muramatsu, T., Suto, Y., Hirai, M., Konishi, T., Hayashi, S., Shigemizu, D., Tsunoda, T., Moriyama, K. y Inazawa, J. (2016). Chromothripsis-like chromosomal rearrangements induced by ionizing radiation using proton microbeam irradiation system. Oncotarget, 7(9), 10182-10192. https://doi.org/10.18632/oncotarget.7186

Nickoloff, J., Sharma, N. y Taylor, L. (2013). Biological consequences of radiation-induced DNA damage: Relevance to radiotherapy. Genes, 11(99). https://doi.org/10.1016/j.clon.2013.06.007

Nikitaki, Z., Nikolov, V., Mavragani, I. V., Mladenov, E., Mangelis, A., Laskaratou, D. A., Fragkoulis, G. I., Hellweg, C. E., Martin, O. A., Emfietzoglou, D., Hatzi, V. I., Terzoudi, G. I., Iliakis, G. y Georgakilas, A. G. (2016). Measurement of complex DNA damage induction and repair in human cellular systems after exposure to ionizing radiations of varying linear energy transfer (LET). Free Radical Research, 50(sup1), S64-S78. https://doi.org/10.1080/10715762.2016.1232484

Okada, K. y Fujisawa, M. (2019). Recovery of spermatogenesis following cancer treatment with cytotoxic chemotherapy and radiotherapy. World Journal of Men's Health, 37(2), 166-174. https://doi.org/10.5534/wjmh.180043

Organismo Internacional de Energía Atómica. (2014). Dosimetría citogenética: Aplicaciones en materia de preparación y respuesta a las emergencias radiológicas. Organismo Internacional de Energía Atómica.

Pajic, J., Jovicic, D. y Milovanovic, A. (2017). Micronuclei as a marker for medical screening of subjects continuously occupationally exposed to low doses of ionizing radiation. Biomarkers, 22(5), 439-445. https://doi.org/10.1080/1354750X.2016.1217934

Palanikumar, L. y Panneerselvam, N. (2011). Micronuclei assay: A potential biomonitoring protocol in occupational exposure studies. Russian Journal of Genetics, 47(9), 1033-1038. https://doi.org/10.1134/S1022795411090146

Purnami, S., Nurhayati, S., Syaifudin, M. y Ramadhani, D. (2017). Biological Dosimetry Using Micronucleus Assay in Simulated Partial-Body Exposure to Ionizing Radiation. Atom Indonesia, 43(2), 75-80.

Rassool, F. V. y Tomkinson, A. E. (2010). Targeting abnormal DNA double strand break repair in cancer. Cellular and Molecular Life Sciences》: CMLS. https://doi.org/10.1007/s00018-010-0493-5

Rawojć, K., Tarnawska, D. M., Miszczyk, J. U., Swakoń, J., Stolarczyk, L. y Rydygier, M. (2015). Application of the micronucleus assay performed by different scorers in case of large-scale radiation accidents. Nukleonika, 60(3), 643-649. https://doi.org/10.1515/nuka-2015-0105

Rodrigues, M. A., Beaton-Green, L. A., Kutzner, B. C. y Wilkins, R. C. (2014). Multi-parameter dose estimations in radiation biodosimetry using the automated cytokinesis-block micronucleus assay with imaging flow cytometry. Cytometry Part A, 85(10), 883-893. https://doi.org/10.1002/cyto.a.22511

Rodrigues, M. A., Probst, C. E., Beaton-Green, L. A. y Wilkins, R. C. (2016). Optimized automated data analysis for the cytokinesis-block micronucleus assay using imaging flow cytometry for high throughput radiation biodosimetry. Cytometry Part A. https://doi.org/10.1002/cyto.a.22887 
Rodrigues, M., Beaton-Green, L., Wilkins, R. y Fenech, M. (2018). The potential for complete automated scoring of the cytokinesis block micronucleus cytome assay using imaging flow cytometry. Mutation Research/Genetic Toxicology and Environmental Mutagenesis, 836, 53-64. https://doi.org/https://doi.org/10.1016/j.mrgentox.2018.05.003

Sabharwal, R., Verma, P., Syed, M., Sharma, T., Subudhi, S., Mohanty, S. y Gupta, S. (2015). Emergence of micronuclei as a genomic biomarker. Indian Journal of Medical and Paediatric Oncology. https://doi.org/10.4103/0971-5851.171541

Sacristán, M. (2015). Modelado de Supervivencia Celular a Radiación Ionizante Basado en la Maximización de la Entropía de Tsallis [Universidad Nacional de Educación a Distancia de España]. http://espacio.uned.es/fez/eserv/bibliuned:master-Ciencias-FMed-

Masacristan/Sacristan_Fernandez_Miguel_Angel_TCI.pdf

Sari-Minodier, I., Orsière, T., Auquier, P., Martin, F. y Botta, A. (2007). Cytogenetic monitoring by use of the micronucleus assay among hospital workers exposed to low doses of ionizing radiation. Mutation Research, 629(2), 111-121. https://doi.org/10.1016/j.mrgentox.2007.01.009

Schipler, A. y Iliakis, G. (2013). DNA double-strand-break complexity levels and their possible contributions to the probability for error-prone processing and repair pathway choice. Nucleic Acids Research, 41(16), 7589-7605. https://doi.org/10.1093/nar/gkt556

Shibai-Ogata, A., Tahara, H., Yamamoto, Y., Fujita, M., Satoh, H., Yuasa, A., Hioki, T. y Kasahara, T. (2014). An automated new technique for scoring the in vivo micronucleus assay with image analysis. Mutagenesis, 29(1), 63-71. https://doi.org/10.1093/mutage/get064

Shibata, A. (2017). Regulation of repair pathway choice at two-ended DNA double-strand breaks. Mutation Research - Fundamental and Molecular Mechanisms of Mutagenesis, 803-805, 51-55. https://doi.org/10.1016/j.mrfmmm.2017.07.011

Sierra, C. (2011). Evaluación del efecto genotóxico de la Radiación lonizante en médicos ortopedistas expuestos laboralmente, en cuatro instituciones de salud en Bogotá, Colombia 2011. Universidad Nacional de Colombia, Bogotá, Colombia.

Sommer, S., Buraczewska, I. y Kruszewski, M. (2020). Micronucleus assay: The state of art, and future directions. International Journal of Molecular Sciences, 21(4). https://doi.org/10.3390/ijms21041534

Strimbu, K. y Tavel, J. (2010). What are biomarkers? Current Opinion in HIV and AIDS, 5(6), 463-466.

Sullivan, J. M., Prasanna, P. G. S., Grace, M. B., Wathen, L. K., Wallace, R. L., Koerner, J. F. y Coleman, C. N. (2013). Assessment of biodosimetry methods for a mass-casualty radiological incident: Medical response and management considerations. Health Physics. https://doi.org/10.1097/HP.0b013e31829cf221

Suto, Y., Akiyama, M., Noda, T. y Hirai, M. (2015). Construction of a cytogenetic dose-response curve for low-dose range gamma-irradiation in human peripheral blood lymphocytes using three-color FISH. Mutation Research - Genetic Toxicology and Environmental Mutagenesis, 794, 32-38. https://doi.org/10.1016/j.mrgentox.2015.10.002 
Tamizh Selvan, G., Chaudhury, N. K. y Venkatachalam, P. (2015). Comparison of results of the manual and automated scoring of micronucleus frequencies in 60Co-irradiated peripheral blood lymphocytes for triage dosimetry. Applied Radiation and Isotopes, 97, 70-77. https://doi.org/10.1016/j.apradiso.2014.12.018

Torres-Bugarín, O., Zavala-Cerna, M. G., Nava, A., Flores-García, A. y Ramos-Ibarra, M. L. (2014). Potential uses, limitations, and basic procedures of micronuclei and nuclear abnormalities in buccal cells. In Disease Markers. https://doi.org/10.1155/2014/956835

Vaiserman, A. M. (2010). Radiation hormesis: Historical perspective and implications for low-dose cancer risk assessment. Dose-Response, 8(2), 172-191. https://doi.org/10.2203/dose-response.09037.Vaiserman

Velickova, N., Milev, M., Ruskovska, T., Petrova, B., Nedeljkovik, B. y Gorgieva, P. (2015). Cytogenetic abnormalities in lymphocytes evaluated with micronucleus assay in medical personnel occupationally exposed to ionizing radiation. Genetika, 47(3), 927-939.

Vral, A., Fenech, M. y Thierens, H. (2011). The micronucleus assay as a biological dosimeter of in vivo ionising radiation exposure. Mutagenesis, 26(1), 11-17. https://doi.org/10.1093/mutage/geq078

Willems, P., August, L., Slabbert, J., Romm, H., Oestreicher, U., Thierens, H. y Vral, A. (2010). Automated micronucleus (MN) scoring for population triage in case of large scale radiation events. International Journal of Radiation Biology. https://doi.org/10.3109/09553000903264481

Zakeri, F. y Hirobe, T. (2010). A cytogenetic approach to the effects of low levels of ionizing radiations on occupationally exposed individuals. European Journal of Radiology, 73(1), 191-195. https://doi.org/10.1016/j.ejrad.2008.10.015

Zhang, X., Ye, C., Sun, F., Wei, W., Hu, B. y Wang, J. (2016). Both complexity and location of DNA damage contribute to cellular senescence induced by ionizing radiation. PLOS ONE, 11(5). https://doi.org/10.1371/journal.pone.0155725 


\section{Población y Salud en Mesoamérica}

¿Quiere publicar en la revista?

Ingrese aquí

O escríbanos:

Revista.ccp@ucr.ac.cr

\section{$1^{\circ}$ Revista}

Electrónica UCR

13 años de liderazgo

Enlace Award, 2007

\section{Cuartil A del}

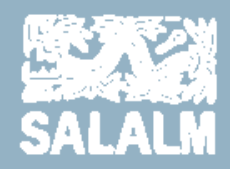

\section{UCR Index}

Población y Salud en Mesoamérica (PSM) es la revista electrónica que cambió el paradigma en el área de las publicaciones científicas electrónicas de la UCR. Logros tales como habersido la primera en obtener sello editorialcomo revista electrónica la posicionan como una de las más visionarias.

\section{Revista PSM es la letra delta mayúscula, el cambio y el futuro.}

Indexada en los catálogos más prestigiosos. Para conocer la lista completa de índices, ingreseaquí.

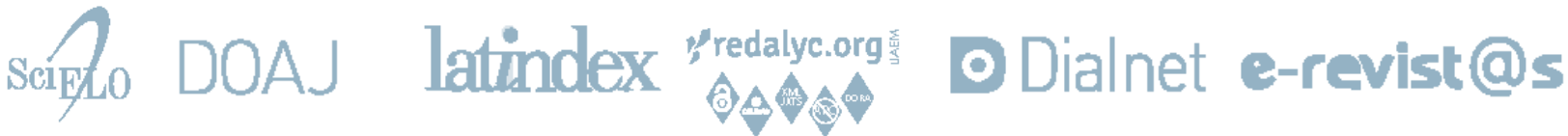

